\section{Fatores associados à não realização do exame de Papanicolaou: um estudo de base populacional no Município de Campinas, São Paulo, Brasil}

\author{
Factors associated with women's failure \\ to submit to Pap smears: a population-based \\ study in Campinas, São Paulo, Brazil
}

Vivian Mae Schmidt Lima Amorim 1 Marilisa Berti de Azevedo Barros 1 Chester Luiz Galvão César ${ }^{2}$ Luana Carandina 3 Moisés Goldbaum 4

\footnotetext{
1 Faculdade de Ciências Médicas, Universidade Estadual de Campinas, Campinas, Brasil.

2 Faculdade de Saúde Pública, Universidade de São Paulo, São Paulo, Brasil.

3 Faculdade de Medicina. Universidade Estadual Paulista, Botucatu, Brasil. 4 Faculdade de Medicina, Universidade de São Paulo São Paulo, Brasil.

Correspondência V. M. S. L. Amorim Departamento de Medicina Preventiva e Social, Faculdade de Ciências Médicas, Universidade Estadual de Campinas. C. P. 6111, Campinas, $S P$ 13083-970, Brasil.
}

\begin{abstract}
This study analyzes the prevalence of non-submittal to Pap smears according to socioeconomic, demographic, and health-related behavioral variables in women 40 years or older in Campinas, São Paulo State. This was a cross-sectional population-based study with a sample of 290 women. Based on multivariate analysis, factors associated with not having Pap smears were: age (40-59 years), racelethnicity (black or mixedrace), and schooling ( $\leq 4$ years). The following reasons were cited for not having Pap smears: considered unnecessary (43.5\%), embarrassment (28.1\%), and barriers related to health services (13.7\%). The Unified National Health System performed $43.2 \%$ of the reported Pap smears. Health services should promote more equitable access to the health care system and improve the quality of care for women, since Pap smears are an effective tool against cervical cancer. The study confirmed that women's failure to obtain Pap smears is associated with social and racial inequality, placing these women at increased risk of cervical cancer.
\end{abstract}

Vaginal Smears; Uterine Cervical Neoplasms; Women's Health

\section{Introdução}

O câncer de colo de útero ocupa uma importante posição como causa de mortalidade, especialmente em países menos desenvolvidos 1 . É um câncer que tem vários fatores de risco identificados e para o qual está disponível um exame eficaz para a detecção precoce: o exame de citologia oncótica, mais conhecido como Papanicolaou 2,3.

A idade avançada, o baixo nível sócio-econômico, pertencer a certos grupos étnicos, não ter cônjuge (solteiras, separadas e viúvas), entre outros, têm sido identificados como fatores associados à não realização do exame de Papanicolaou 4,5. A limitação do acesso aos serviços de saúde, por barreiras sócio-econômicas, culturais, e geográficas também se apresenta como responsável pela baixa cobertura dos exames de citologia oncótica, sendo um problema a ser enfrentado pelos gestores do programa de controle do câncer de colo de útero 6,7,8.

A realização do exame de Papanicolaou é recomendada, por organizações nacionais e internacionais de saúde, para as mulheres que já tenham iniciado a atividade sexual. No Brasil, desde 1988, o Ministério da Saúde (MS) segue a recomendação da Organização Mundial da Saúde (OMS), que propõe a realização do exame a cada três anos, após dois controles anuais consecutivos negativos para mulheres com até 59 anos de idade 9 . 
Apesar da implantação do programa da mulher e da ampliação da cobertura do exame de Papanicolaou, não tem havido redução das taxas de incidência e de mortalidade do câncer de colo de útero, no Brasil, tendo a taxa de mortalidade aumentado nas últimas décadas de 3,44/100 mil mulheres, em 1979, para 4,59/100 mil, em 2000 10. No Estado de São Paulo, a taxa bruta de mortalidade pelo câncer de colo de útero tem se mostrado estável desde a década de 70, com valores próximos a 4,2/100 mil, tendo havido um relativo deslocamento dos óbitos para as idades acima de 70 anos 3 .

Na cidade de Campinas, dados do Registro de Câncer de Base Populacional mostram que, entre 1991 e 1995, a taxa ajustada da incidência do câncer do colo do útero foi de 17,3/100 mil, e que o aumento de incidência da doença ocorre a partir dos 40 anos de idade 11. Em 2004, o câncer de colo de útero representou $5 \%$ das mortes femininas por neoplasias em Campinas, com uma taxa de aproximadamente sete óbitos por $100 \mathrm{mil}$ em mulheres de 25 anos ou mais 12 .

Considerando que o câncer de colo de útero é uma neoplasia que apresenta elevada taxa de incidência e de mortalidade, passível de detecção precoce e de ser curável dependendo do estágio em que é detectado 3 , buscou-se com este estudo analisar os fatores associados à não realização do exame de Papanicolaou no $\mathrm{Mu}$ nicípio de Campinas, esperando que os resultados possam contribuir para o aprimoramento das ações de prevenção e controle do câncer do colo de útero.

\section{Material e métodos}

O estudo realizado foi do tipo transversal, de base populacional, utilizando-se os dados do Inquérito Multicêntrico de Saúde no Estado de São Paulo (ISA-SP), realizado entre 2001-2002, em quatro áreas do Estado de São Paulo: municípios de Campinas, Botucatu, Itapecerica da Serra/Embu/Taboão da Serra e distrito do Butantã, no Município de São Paulo 13 .

Para a obtenção das amostras do Inquérito ISA-SP, os setores censitários dos municípios participantes foram agrupados em três estratos segundo o percentual de chefes de família com nível universitário: menos de $5 \%$, de 5 a $25 \%$ e com $25 \%$ ou mais. Essa estratificação foi feita para aumentar a probabilidade de que indivíduos pertencentes aos estratos de maior nível sócio-econômico da população fossem incluídos na amostra. Foram sorteados dez setores censitários de cada estrato, e em cada se- tor foram sorteados os domicílios e selecionados os indivíduos que seriam entrevistados. Para atender ao objetivo de obter estimativas para subpopulações, definidas como domínios, foram considerados os seguintes grupos de sexo e idade: menores de 1 ano; de 1 a 11 anos; homens de 12 a19 anos; mulheres de 12 a 19; homens de 20 a 59; mulheres de 20 a 59; homens de 60 anos ou mais e mulheres de 60 anos ou mais de idade.

O tamanho mínimo da amostra foi estimado em 200 indivíduos para cada um dos domínios, tendo como base a estimativa de uma proporção de $50 \%$, com erro máximo de $7 \%$, erro tipo a de $5 \%$ e efeito de delineamento de 2 . Considerando a possibilidade de $20 \%$ de perda (casa fechada e recusas), foram sorteados 250 indivíduos para cada domínio.

As informações foram obtidas por meio de questionário estruturado em 19 blocos temáticos, com a maioria das questões fechadas, aplicado diretamente à pessoa sorteada por entrevistadores treinados e supervisionados durante toda a duração do inquérito. Questionários com informação incompleta ou inconsistente voltavam a campo para serem complementados. O estudo piloto foi realizado em um dos municípios do estudo. Não foram incluídos como entrevistadores pessoas com formação na área de saúde, para evitar interferência na interpretação das respostas. A confidenciabilidade das informações foi garantida.

Para o presente estudo, foram utilizados os dados das mulheres com 40 anos ou mais, residentes em Campinas, que pertenciam ao domínio de 20 a 59 anos $(\mathrm{n}=94)$ e ao domínio de 60 anos ou mais ( $n=222$ ), compondo uma amostra de 316 mulheres. Como não foi possível realizar a entrevista em 26 casos por motivo de recusa, o que representou uma perda de $8,2 \%$, a amostra estudada foi de 290 mulheres. Para a escolha da faixa etária a ser estudada, 40 anos ou mais, foram considerados os dados nacionais e locais que apontam o aumento da incidência do câncer do colo do útero a partir dos 40 anos de idade 10,12 .

Os blocos do questionário com as questões de interesse específico para este trabalho foram os referentes às características sócio-econômicas do entrevistado e do chefe da família, às doenças crônicas auto-referidas, deficiência física, utilização de serviços, exames preventivos, estilo de vida, saúde emocional e autoavaliação em saúde.

A variável dependente foi definida como não realização do exame de Papanicolaou. Para as mulheres entre 40 e 59 anos de idade considerou-se a não realização do exame nos últimos 
três anos anteriores à entrevista e, para as mulheres com idade igual ou superior a 60 anos, o fato de nunca terem realizado o exame.

As variáveis independentes analisadas neste estudo foram:

Variáveis sócio-econômicas e demográficas: idade, cor/raça (auto-referida), naturalidade, situação conjugal, escolaridade, posição na família, renda familiar mensal per capita, situação ocupacional, religião, número de pessoas no domicílio e posse de bens duráveis. Para o cálculo da renda familiar per capita foram considerados os valores dos salários mínimos vigentes no país por ocasião da realização do inquérito: salário mínimo de R\$ 151,00 (março a abril de 2001), $\mathrm{R} \$ 180,00$ (maio de 2001 a março de 2002) e de $\mathrm{R} \$ 200,00$ (abril a setembro de 2002).

Variáveis de comportamentos relacionados à saúde: atividade física em contexto de lazer e hábito de fumar (fumantes, ex-fumantes e nãofumantes).

Variáveis relacionadas ao estado de saúde: doenças crônicas referidas, limitação física, presença de "transtorno mental comum" avaliada pelo Self Reporting Questionnaire (SRQ-20), presença de obesidade utilizando-se o Índice de Massa Corporal (IMC $=\mathrm{kg} / \mathrm{m}^{2}$ ) calculado com base no peso e altura auto-referidos. As mulheres com IMC $\geq 25$ foram classificadas como tendo sobrepeso ou obesidade. Para as mulheres com 60 anos ou mais de idade também foi analisada, porque apenas para elas estava disponível, a variável auto-avaliação da saúde, por meio de pergunta fechada: "Em geral como a senhora diria que sua saúde é: excelente, muito boa, boa, ruim, muito ruim?".

Variáveis relacionadas à realização de práticas preventivas para o câncer de mama: autoexame das mamas (independente da freqüência da realização), exame clínico das mamas no último ano e mamografia nos últimos dois anos.

Variáveis relativas a outras práticas preventivas concomitantes: vacinação contra gripe para mulheres com 60 anos ou mais.

Os dados foram digitados em programa Epi Info 6.04b (Centers for Disease Control and Prevention, Atlanta, Estados Unidos), e submetidos à análise de consistência. Para as análises estatísticas foi utilizado o programa Stata 7.0 (Stata Corporation, College Station, Estados Unidos), o que possibilitou levar em consideração as variáveis do plano de amostragem: estratos, conglomerados, ponderação e o efeito do delineamento. As análises bivariadas incluíram estimativas de prevalência e intervalos de $95 \%$ de confiança (IC95\%) da não realização do exame de Papanicolaou segundo as variáveis independentes. Foram estimadas razões de prevalência e IC95\% e análise ajustada por meio de regressão de Poisson, que é uma medida mais conservadora e interpretável do que a razão das chances 14 . No modelo múltiplo foram consideradas as variáveis que tiveram valor de $p<0,20$, na análise bivariada, permanecendo no modelo se $\mathrm{p}<0,05$.

Este projeto foi aprovado pela Comissão de Ética da Faculdade de Ciências Médicas, Universidade Estadual de Campinas, sob parecer $369 / 2000$.

\section{Resultados}

Dentre as 290 mulheres com 40 anos ou mais de idade incluídas no estudo, 71,7\% tinham 60 anos ou mais. Em relação à cor/raça, 81,9\% referiramse como brancas. Quanto ao estado civil, $42,3 \%$ eram casadas ou tinha companheiro. A maioria tinha até quatro anos de escolaridade $(64,4 \%) \mathrm{e}$ $37,2 \%$ exerciam a chefia da família. Em relação à realização do exame de Papanicolaou, 16,7\% das mulheres não haviam realizado o exame nos três anos que antecederam a entrevista (Tabela 1), sendo que $8,5 \%$ das mulheres entre 40 e 59 anos e $11,2 \%$ com 60 anos ou mais referiram que nunca haviam realizado um exame de Papanicolaou.

A Tabela 1 mostra que a não realização do exame de Papanicolaou mostrou-se associada a diversas variáveis demográficas e sócio-econômicas, apresentando prevalências de não realização do exame significativamente mais elevadas as mulheres com idade entre 40 e 59 anos, com até quatro anos de escolaridade, com renda mensal familiar per capita menor ou igual a quatro salários mínimos, com a posse de até nove bens duráveis, que se auto-referiram pretas/pardas e que moravam em domicílios com cinco ou mais pessoas.

Observou-se que, entre os comportamentos relacionados à saúde e à morbidade, a não realização do exame de Papanicolaou associou-se de forma estatisticamente significante apenas com a auto-avaliação da saúde (Tabela 2).

Os resultados da análise de regressão múltipla de Poisson (Tabela 3) apontam que a não realização do exame de Papanicolaou foi significativamente mais freqüente nas mulheres com escolaridade de até quatro anos, que se auto-referiram pretas/pardas e com idade entre 40 e 59 anos.

A Tabela 4 revela que a não realização do exame do Papanicolaou foi significativamente mais freqüente nas mulheres que não realizaram outras práticas preventivas e de cuidados à saúde como o auto-exame mensal das mamas, exame clínico das mamas no último ano e mamografia nos últimos dois anos. 
Prevalência da não realização do exame de Papanicolaou segundo variáveis sócio-econômicas e demográficas, em mulheres de 40 anos ou mais. Campinas, São Paulo, Brasil, 2001-2002.

\begin{tabular}{|c|c|c|c|}
\hline Variáveis & $N=290$ & $\begin{array}{l}\text { Prevalência } \\
\text { (IC95\%) }\end{array}$ & $\begin{array}{c}\text { Razão de prevalências } \\
\text { ajustadas pela idade } \\
\text { (IC95\%) }\end{array}$ \\
\hline Idade (anos) & & $0,0496^{*}$ & \\
\hline $40-59$ & 82 & $19,4(10,7-32,5)$ & $1,73(1,01-2,97)$ \\
\hline $60 \mathrm{ou}+$ & 208 & $11,2(7,8-15,6)$ & 1,00 \\
\hline Total & 290 & $16,7(10,2-26,2)$ & \\
\hline Cor/Raça** (auto-referida) & & $0,0059 *$ & \\
\hline Branca & 236 & $10,9(5,8-19,7)$ & 1,00 \\
\hline Preta/Parda & 52 & $36,3(17,5-60,0)$ & $3,22(1,43-7,26)$ \\
\hline Total & 288 & & \\
\hline Naturalidade & & $0,1615^{\star}$ & \\
\hline Estado de São Paulo & 188 & $12,6(6,7-22,6)$ & 1,00 \\
\hline Outros Estados & 101 & $21,6(11,4-37,3)$ & $1,58(0,72-3,47)$ \\
\hline Total & 289 & & \\
\hline Situação conjugal & & $0,2590 *$ & \\
\hline Com companheiro & 139 & $14,7(7,7-24,5)$ & 1,00 \\
\hline Sem companheiro & 151 & $20,6(11,4-33,4)$ & $1,62(0,84-3,13)$ \\
\hline Total & 290 & & \\
\hline Religião & & $0,4476^{*}$ & \\
\hline Católica & 218 & $18,3(10,9-29,0)$ & $1,47(0,55-3,94)$ \\
\hline Outras & 72 & $12,8(4,9-29,7)$ & 1,00 \\
\hline Total & 290 & & \\
\hline Situação na família & & $0,9318^{\star}$ & \\
\hline Chefe & 108 & $16,3(7,4-32,1)$ & 1,00 \\
\hline Não chefe & 182 & $16,9(9,3-28,9)$ & $0,97(0,38-2,44)$ \\
\hline Total & 290 & & \\
\hline Pessoas no domicílio & & $0,0103^{\star}$ & \\
\hline $1-4$ & 220 & $11,1(6,2-19,2)$ & 1,00 \\
\hline 5 ou + & 70 & $27,8(15,2-45,3)$ & $2,33(1,12-4,83)$ \\
\hline Total & 290 & & \\
\hline Escolaridade (anos) & & $0,0416^{*}$ & \\
\hline $0-4$ & 186 & $23,7(13,6-38,1)$ & $3,12(1,15-8,42)$ \\
\hline 5 ou + & 103 & $9,5(4,1-20,8)$ & 1,00 \\
\hline Total & 289 & & \\
\hline Renda familiar per capita (salários mínimos) & & $0,0103^{\star}$ & \\
\hline$\leq 4$ & 210 & $21,5(12,8-33,8)$ & $4,22(1,32-13,49)$ \\
\hline$>4$ & 80 & $5,3(1,7-15,3)$ & 1,00 \\
\hline Total & 290 & & \\
\hline Situação ocupacional & & $0,3432^{\star}$ & \\
\hline Trabalha & 74 & $20,6(9,1-28,9)$ & $1,28(0,47-3,45)$ \\
\hline Não trabalha & 216 & $13,6(8,5-21,0)$ & 1,00 \\
\hline Total & 290 & & \\
\hline Número de bens duráveis & & $0,0132^{\star}$ & \\
\hline $1-9$ & 185 & $23,2(4,1-35,9)$ & $3,19(1,30-784)$ \\
\hline 10 ou + & 105 & $8,0(3,2-18,4)$ & 1,00 \\
\hline Total & 290 & & \\
\hline
\end{tabular}

$\mathrm{N}$ = número de indivíduos na amostra não ponderada;

* Valor $p$ do teste $\chi^{2}$;

** Excluídas duas da cor amarela. 
Prevalência da não realização do exame de Papanicolaou segundo comportamentos relacionados à saúde e morbidade, em mulheres de 40 anos ou mais. Campinas, São Paulo, Brasil, 2001-2002.

\begin{tabular}{|c|c|c|c|}
\hline Variáveis & $N=290$ & $\begin{array}{l}\text { Prevalência } \\
\text { (IC95\%) }\end{array}$ & $\begin{array}{c}\text { Razão de prevalências } \\
\text { ajustadas pela idade } \\
\text { (IC95\%) }\end{array}$ \\
\hline Hábito de fumar & & $0,3478^{*}$ & \\
\hline Ex-fumantes & 50 & $7.8(2,7-20,3)$ & 1,00 \\
\hline Fumantes & 41 & $17,8(7,5-36,7)$ & $2,02(0,54-7,53)$ \\
\hline Não-fumantes & 199 & $19,2(10,3-32,8)$ & $2,65(0,87-8,06)$ \\
\hline Total & 290 & & \\
\hline Prática de exercícios físicos & & $0,3535^{\star}$ & \\
\hline Sim & 81 & $12,6(5,7-25,4)$ & 1,00 \\
\hline Não & 209 & $18,5(10,5-30,5)$ & $1,52(0,64-3,61)$ \\
\hline Total & 290 & & \\
\hline IMC & & $0,5500^{*}$ & \\
\hline$<25$ & 117 & $14,0(7,1-25,8)$ & 1,00 \\
\hline$\geq 25$ & 138 & $17,3(8,7-31,6)$ & $1,27(0,61-2,64)$ \\
\hline Total & 265 & & \\
\hline Número de doenças crônicas referidas & & $0,3348^{*}$ & \\
\hline $0-3$ & 187 & $18,2(11,2-18,2)$ & $1,38(0,57-3,30)$ \\
\hline 4 ou + & 94 & $12,1(4,8-27,4)$ & 1,00 \\
\hline Total & 281 & & \\
\hline Limitação física referida & & $0,6964^{*}$ & \\
\hline Não & 213 & $16,2(9,8-25,6)$ & 1,00 \\
\hline Sim & 77 & $18,8(8,0-38,1)$ & $1,30(0,57-2,95)$ \\
\hline Total & 290 & & \\
\hline Transtorno mental comum & & $0,6394^{*}$ & \\
\hline Não & 204 & $15,5(8,1-27,4)$ & 1,00 \\
\hline $\operatorname{Sim}$ & 71 & $19,1(8,7-36,9)$ & $1,26(0,50-3,18)$ \\
\hline Total & 274 & & \\
\hline Auto-avaliação da saúde & & $0,0387^{\star}$ & \\
\hline Excelente/Muito boa & 59 & $4,9(1,7-13,5)$ & 1,00 \\
\hline Boa & 118 & $11,1(6,4-18,5)$ & $2,26(0,64-7,91)$ \\
\hline Ruim/Muito ruim & 23 & $26,1(10,3-52,0)$ & $5,31(1,35-20,89)$ \\
\hline Total & 200 & & \\
\hline
\end{tabular}

$\mathrm{N}$ = número de indivíduos na amostra não ponderada;

* Valor $p$ do teste $\chi^{2}$;

** Mulheres com 60 anos ou mais.

Entre os motivos apontados para a não realização do exame, foi referido com maior freqüência o fato da mulher achar que não é necessário realizá-lo (43,5\%), seguido pelo motivo de considerá-lo um "exame embaraçoso" $(28,1 \%)$. O não conhecimento do exame foi referido por $5,7 \%$ das mulheres, e a dificuldade em marcar o exame por $13,7 \%$. Outros motivos foram alegados por $7,2 \%$ e 1,8\% não responderam. Das mulheres que realizaram o exame de
Papanicolaou, 43,2\% relataram tê-lo realizado em serviços do SUS (dados não apresentados em tabelas).

\section{Discussão}

O ISA-SP 13 abrangeu três áreas temáticas: estilo de vida, situação de saúde e o uso do serviço de saúde. Neste último, está inserido o bloco 
Tabela 3

Modelo de regressão múltipla de Poisson para a não realização do exame de Papanicolaou em mulheres com 40 anos ou mais. Campinas, São Paulo, Brasil, 2001-2002.

\begin{tabular}{|c|c|c|c|c|c|}
\hline Variáveis & $\mathbf{N}$ & $\begin{array}{c}\text { Razão de } \\
\text { prevalências* }\end{array}$ & Valor de $p$ & IC95\% & $\begin{array}{c}\text { Efeito de } \\
\text { delineamento }\end{array}$ \\
\hline \multicolumn{6}{|l|}{ Idade (anos) } \\
\hline $40-59$ & 82 & 2,01 & 0,013 & $1,17-3,45$ & 0,62 \\
\hline $60 \mathrm{ou}+$ & 208 & 1,00 & & & \\
\hline Total & 290 & & & & \\
\hline \multicolumn{6}{|c|}{ Escolaridade (anos) } \\
\hline 5 ou + & 103 & 1,00 & & & \\
\hline $0-4$ & 186 & 2,77 & 0,030 & $1,07-7,12$ & 2,01 \\
\hline Total & 289 & & & & \\
\hline \multicolumn{6}{|l|}{ Cor/Raça } \\
\hline Branca & 236 & 1,00 & & & \\
\hline Negra/Parda & 52 & 2,35 & 0,023 & $1,13-4,87$ & 2,20 \\
\hline Total & 288 & & & & \\
\hline
\end{tabular}

* Ajustadas pelo modelo de Poisson para as demais variáveis do modelo.

Tabela 4

Prevalência da não realização do exame de Papanicolaou segundo outras práticas preventivas e de cuidados à saúde, em mulheres de 40 anos ou mais. Campinas, São Paulo, Brasil, 2001-2002.

\begin{tabular}{|c|c|c|c|}
\hline Variáveis & $\mathbf{N}$ & $\begin{array}{l}\text { Prevalência } \\
\text { (IC95\%) }\end{array}$ & $\begin{array}{c}\text { Razão de prevalências } \\
\text { ajustadas para idade } \\
\text { (IC95\%) }\end{array}$ \\
\hline $\begin{array}{l}\text { Prática do auto-exame (independente } \\
\text { da freqüência) }\end{array}$ & & $0,0215^{\star}$ & \\
\hline Sim & 204 & $12,5(6,6-22,4)$ & 1,00 \\
\hline Não & 86 & $28,8(16,2-43,8)$ & $2,23(1,14-4,74)$ \\
\hline Total & 290 & & \\
\hline Exame clínico das mamas (no último ano) & & $0,0000^{*}$ & \\
\hline Sim & 164 & $3,8(1,3-10,6)$ & 1,00 \\
\hline Não & 126 & $37,7(23,0-55,1)$ & $10,90(3,48-34,10)$ \\
\hline Total & 290 & & \\
\hline Realização da mamografia (nos últimos dois anos) & & $0,0003^{*}$ & \\
\hline Sim & 160 & $6,0(2,2-15,3)$ & 1,00 \\
\hline Não & 130 & $31,3(19,6-45,8)$ & $5,38(2,03-14,23)$ \\
\hline Total & 290 & & \\
\hline Vacina de gripe (mulheres com 60 anos ou mais) & & $0,1159 *$ & \\
\hline Sim & 128 & $8,8(4,8-15,4)$ & 1,00 \\
\hline Não & 78 & $15,4(10,1-15,8)$ & $1,75(0,84-3,63)$ \\
\hline Total & 206 & & \\
\hline
\end{tabular}

$\mathrm{N}$ = número de indivíduos na amostra não ponderada;

* Valor $p$ do teste $\chi^{2}$. 
temático que inclui a realização de alguns exames preventivos, entre eles o exame de Papanicolaou, que é o objeto deste artigo.

Os principais resultados do presente estudo foram: a verificação de uma cobertura do exame de Papanicolaou julgada aquém das possibilidades dos serviços de saúde existentes no município; a presença de significativas desigualdades sociais e raciais na realização do exame; o desconhecimento sobre a importância do Papanicolaou e a referência do "constrangimento" pelas mulheres que nunca fizeram o exame; e uma grande cobertura da realização do exame pelos serviços de saúde privados.

Uma das limitações deste estudo é o fato da informação sobre a realização do exame de $\mathrm{Pa}$ panicolaou ter sido referida e não checada em prontuários ou registros médicos, podendo, portanto, ser sujeita a vieses de memória e de informação. A entrevistada pode ter-se equivocado quanto ao tempo decorrido desde a realização do último exame, ou ainda dizer que realizou o exame nos últimos três anos por considerar esta conduta a adequada e esperada. Estudo realizado nos Estados Unidos observou uma alta correlação entre os dados auto-referidos sobre as práticas preventivas para vários tipos de câncer, inclusive do colo do útero, e os dados registrados nos prontuários das pessoas entrevistadas, apontando as entrevistas como um método confiável e menos dispendioso para a obtenção desse tipo de informação 15 . Vários estudos nacionais 6,16,17,18 e internacionais 19,20 têm utilizado também informação referida sobre a realização do exame de citologia oncótica cervical, o que tem sido considerado um procedimento válido e de menor custo.

Outra limitação deste estudo decorre da utilização de dados de inquérito de saúde mais amplo, que não se voltou especificamente para as práticas preventivas do câncer de colo do útero, de forma que algumas informações importantes deixaram de ser levantadas, como conhecimento e atitude em relação ao exame, se as entrevistadas eram ou não sexualmente ativas e se as mulheres que referiram ter realizado o Papanicolaou tinham o conhecimento do resultado do exame.

O desenho transversal do estudo limita, também, a possibilidade de interpretar as associações encontradas como derivadas de relações de causa-efeito.

A presente pesquisa possibilitou verificar que $83,3 \%$ (IC95\%: 73,8-89,8\%) das mulheres com 40 anos ou mais de idade, residentes em Campinas, realizaram o exame de Papanicolaou, de acordo com o critério para a sua faixa etária. Para as mulheres da faixa etária de 40 a 59 anos, o resultado encontrado $(80,6 \%)$ é semelhante aos porcentuais verificados no Município de São Paulo, onde foi observado que $79,4 \%$ das mulheres de 35 a 59 anos haviam realizado pelo menos um exame de Papanicolaou nos 3 anos que antecederam a entrevista 16 . O percentual de Campinas é menor do que o observado em Vitória, Espírito Santo (92,3\%) e maior do que o observado em João Pessoa, Paraíba $(74,6 \%) 16$. Considerando a estrutura e a organização da rede de serviços de saúde do Município de Campinas, seria esperada cobertura do exame de Papanicolaou superior à observada, por exemplo, no Município de São Paulo.

Também foi observado que $8,5 \%$ das mulheres de 40 a 59 anos e $11,2 \%$ das de 60 anos ou mais nunca haviam realizado um exame de Papanicolaou. Mulheres com 60 anos ou mais de idade, por não estarem mais em idade fértil, tendem a deixar de realizar consultas ginecológicas, afastando-se das práticas preventivas para o câncer de colo uterino exatamente quando a incidência da doença aumenta 21 . Esse segmento de mulheres mais idosas demanda com freqüência os serviços de saúde em decorrência de outras morbidades, mas essas demandas não estariam sendo adequadamente aproveitadas para a realização do exame de Papanicolaou, o que ocorreria se estivessem sendo contempladas no contexto de uma efetiva atenção integral à saúde.

Os principais motivos referidos pelas mulheres que nunca realizaram o exame de Papanicolaou em Campinas são semelhantes aos relatados em estudo realizado no Município de São Paulo 6. A falta de compreensão da importância da realização do exame de Papanicolaou, por um segmento de mulheres, constitui um desafio para os serviços de saúde, pois tem limitado o acesso ao rastreamento do câncer de colo de útero principalmente daquelas consideradas de maior risco 6,19. Para superar a questão do constrangimento, os serviços de saúde vêm desenvolvendo estratégias para a inclusão dos subgrupos mais vulneráveis. Uma das medidas que vêm sendo adotadas pelos serviços de saúde do SUS é a implantação de protocolos de atendimento de enfermagem para a saúde da mulher, onde a enfermeira, respaldada pela lei do exercício profissional 22 , pode executar a coleta do esfregaço e a inspeção do colo uterino, e dessa forma ampliar a oferta do exame. O serviço da rede municipal de saúde de Campinas tem adotado essa prática, como forma de melhorar a cobertura do exame.

Em relação à idade, as mulheres de 40 e 59 anos apresentaram maior prevalência de não realização do exame de Papanicolaou em com- 
paração às de 60 anos ou mais, o que decorreu da utilização de critérios diferentes de definição de não realização do exame para as duas faixas etárias.

As mulheres de cor auto-referida como preta ou parda apresentaram maior prevalência de não realização do exame de Papanicolaou, achado que persiste mesmo após ajuste para idade e escolaridade, apontando a existência de desigualdade racial quanto ao acesso ao exame de citologia oncótica cervical. Esse achado é semelhante ao encontrado em dois outros trabalhos nacionais 17,23 , confirmando, assim, a existência de desigualdade racial no acesso às atividades do programa de detecção precoce do câncer do colo do útero. Discriminação racial tem sido também observada quanto à assistência prestada durante o ciclo gravídicopuerperal, com as mulheres pretas e pardas tendo menor acesso ao pré-natal, sendo menos aceitas na primeira maternidade procurada $\mathrm{e}$ recebendo com menor freqüência analgesia durante a realização do parto 24 .

Quanto à escolaridade, a não realização do exame de Papanicolaou foi mais freqüente nas mulheres com menor número de anos de estudo, sendo este achado semelhante ao encontrado em pesquisas realizadas em outros municípios brasileiros 6,17 e em outros países 4,7,19,20. Também apareceram associados à não realização do exame de Papanicolaou, na análise bivariada, outros indicadores de nível sócio-econômico, como ter menor renda familiar mensal per capita e posse de menor número bens duráveis. A literatura tem relatado que à medida que diminui o nível sócio-econômico, aumenta significativamente a prevalência de mulheres não cobertas pelo exame do Papanicolaou 6,18,19,23. Essa discriminação social indica a necessidade de intervenção mais efetiva por parte dos serviços públicos de saúde, pois o segmento mais vulnerável da população é o que mais depende dos serviços do SUS. No Município de Campinas, foi observado que a mortalidade por câncer do colo do útero apresenta um risco quatro vezes maior nas mulheres que residem na área urbana de pior nível social, relativamente às moradoras na área de melhor nível 12, apesar da existência de uma rede de saúde bem estruturada e que estaria garantindo um grau razoável de acesso ao diagnóstico e tratamento do câncer do colo do útero.

Em relação à concomitância de outras práticas preventivas, a não realização do exame do Papanicolaou foi mais freqüente nas mulheres que não realizam o auto-exame mensal das mamas, o exame físico anual das mamas e a mamografia. Esses resultados eram esperados con- siderando que o exame clínico das mamas, a solicitação da mamografia e a realização do exame de Papanicolaou, fazem parte do atendimento integral da mulher prestado durante a consulta médica ginecológica e que, na ausência desta consulta, a realização de todos esses procedimentos fica comprometida. Pressupõese que as mulheres que procuram mais os serviços de saúde para a consulta ginecológica, também estariam mais expostas a estímulos e orientação para o auto-exame das mamas, visto que a orientação para essa prática também é parte integrante da consulta ginecológica 25 .

Outro achado relevante do presente estudo é que os serviços privados foram responsáveis por 56,8\% dos exames de Papanicolaou realizados, enquanto os serviços do SUS responderam por $43,2 \%$. Este percentual é muito próximo ao encontrado no Município de São Paulo, no qual $57 \%$ das mulheres que realizaram o exame de Papanicolaou nos três anos que antecederam a entrevista o fizeram em serviços privados 15 . O percentual que o setor privado de Campinas cobre em relação ao exame de Papanicolaou é ainda superior ao observado em outros atendimentos da saúde da mulher, como pré-natal e parto em que o setor privado cobre, respectivamente, $46,8 \%$ e $41,9 \% 26$.

O Programa Saúde da Família, que vem sendo adotado no Brasil como modelo de atenção básica de saúde, pode contribuir para a superação das barreiras existentes à realização do exame de Papanicolaou identificando e captando, pela atuação dos agentes de saúde, as mulheres que deixam de realizar o exame. Esse programa tem como princípio garantir o acesso à atenção básica, a criação de vínculo entre a clientela e a equipe de saúde e a integralidade do atendimento. Como resultado dessas ações espera-se a promoção do conhecimento, a valorização e a incorporação, por parte da população, das práticas preventivas não só para o câncer de colo de útero, como também para outras morbidades 19 .

Pode-se concluir que os achados deste estudo, apontando a existência de significativas desigualdades sócio-econômicas e raciais quanto à realização do exame de Papanicolaou, reforçam a necessidade de intervenções que visem à promoção da eqüidade. Os motivos alegados pelas mulheres para a não realização do exame remetem à necessidade dos serviços de saúde serem mais efetivos nas práticas educativas e em estratégias que minimizem a não cobertura do exame, sobretudo dos grupos em maior vulnerabilidade social e dependentes do atendimento do SUS. 


\section{Resumo}

O objetivo deste estudo foi analisar a prevalência da não realização do exame de Papanicolaou segundo variáveis sócio-econômicas, demográficas e de comportamentos relacionados à saúde, em mulheres com 40 anos ou mais de idade, residentes no Município Campinas, São Paulo, Brasil. O estudo foi do tipo transversal, de base populacional em uma amostra de 290 mulheres. Os fatores associados à não realização do Papanicolaou, encontrados na análise multivariada, foram: ter de 40 a 59 anos, ser pretalparda, ter escolaridade de até 4 anos. Entre os motivos alegados por quem nunca realizou o Papanicolaou destacam-se: achar desnecessário (43,5\%), sentir vergonha $(28,1 \%) e$ $13,7 \%$ por dificuldades relacionadas aos serviços. $O$ SUS foi responsável por 43,2\% dos exames de Papanicolaou realizados. Verificou-se a existência de discriminação racial e social na realização do exame, o que enfatiza a necessidade de intervenções que garantam melhor cobertura e atenção às mulheres mais vulneráveis à incidência e mortalidade por câncer do colo do útero.

Esfregaço Vaginal; Neoplasia do Colo do Útero; Saúde da Mulher

\section{Colaboradores}

V. M. S. L. Amorim realizou a proposta do artigo, a revisão da literatura, a análise dos dados e a redação do artigo. M. B. A. Barros orientou a proposta do artigo, a análise dos dados e a redação do artigo. M. B. A. Barros, C. L. G. César, L. Carandina, M. Goldbaum desenvolveram o projeto Inquérito Multicêntrico de Saúde no Estádio de São Paulo, elaboraram os instrumentos, coordenaram a pesquisa de campo e contribuíram na revisão do artigo.

\section{Agradecimentos}

À Fundação de Amparo à Pesquisa do Estado de São Paulo - Programa de Políticas Públicas, processo no 88/14099-7, e à Secretaria Estadual de São Paulo, pelo financiamento do Inquérito Multicêntrico de Saúde no Estádio de São Paulo. À Secretaria de Vigilância em Saúde do Ministério da Saúde pelo suporte à análise através do Centro Colaborador em Análise de Situação de Saúde, Faculdade de Ciências Médicas, Universidade Estadual de Campinas.

\section{Referências}

1. Todd RW, Shafi M. Invasive cervical cancer. Current Obstet Gynaecol 2004; 14:200-6.

2. Gardner E. Cervical cancer: disparities in screening, treatment, and survival. Cancer Epidemiol Biomarkers Prev 2003; 12:242-7.

3. Fonseca LAM, Ramacciotti AS, Eluf Neto J. Tendência da mortalidade por câncer de útero no Município de São Paulo entre 1980 e 1999. Cad Saúde Pública 2004; 20:136-42.

4. Coughlin SS, Uhler RJ. Breast and cervical screening practices among asian and pacific islander women in the United States, 1994-1997. Cancer Epidemiol Biomarkers Prev 2000; 9:597-603.

5. Abraido-Lanza AF, Chao MT, Gammon MD. Breast and cervical cancer screening among latinas and non latinas whites. Am J Public Health 2004; 94 : 1393-8.

6. Pinho AA, França Junior I, Schraiber LB, D'Oliveira AFPL. Cobertura e motivos para a realização ou não do teste de Papanicolaou no Município de São Paulo. Cad Saúde Pública 2003; 19 Suppl 2:S303-13.

7. Claeys P, Gonzalez C, Page H, Bello RE, Temmerman M. Determinants of cervical screening in poor area: results of a population-based survey in Rivas, Nicaragua. Trop Med Int Health 2002; 7:93541 .

8. Selvin E, Brett KM. Breast and cervical cancer screening: sociodemographic predictors among white, black, and hispanic woman. Am J Public Health 2003; 94:618-23.

9. Instituto Nacional de Câncer. Viva mulher - programa de controle do câncer do colo do útero e de mama: periodicidade de realização do exame preventivo do câncer do colo de útero. http://www. inca.gov.br (acessado em 03/Set/2004).

10. Instituto Nacional de Câncer. Estimativa da incidência e mortalidade por câncer no Brasil - 2002. http://www.inca.gov.br (acessado em 13/Set/2004).

11. Instituto Nacional de Câncer. Câncer no Brasil dados dos registros de base populacional. http:// www.inca.gov.br (acessado em 05/Ago/2005).

12. Secretária Municipal de Saúde/Universidade Estadual de Campinas. Mortalidade em Campinas. Boletim no 36, julho a dezembro de 2004. Mortalidade por câncer de mama e colo de útero. http:// www.campinas.sp.gov.br/saúde (acessado em 10/ Fev/2006).

13. César LGC, Carandina L, Alves MCGP, Barros MBA, Goldbaum M. Saúde e condição de vida em São Paulo. Inquérito Multicêntrico de Saúde no Estado de São Paulo - ISA-SP. São Paulo: Faculdade de Saúde Pública, Universidade de São Paulo; 2005.

14. Barros AJ, Hirata VN. Alternatives for logistic regression in cross-sectional studies: an empirical comparison of models that directly estimative the prevalence ratio. BMC Med Res Methodol 2003; 3:21.

15. Instituto Nacional de Câncer. Inquérito domiciliar sobre comportamento de risco e morbidade referida de doenças e agravos não transmissíveis: Brasil, 15 capitais e Distrito Federal, 2002-2003. Rio de Janeiro: Instituto Nacional de Câncer; 2004. 
16. Cesar JA, Horta BL, Gomes G, Houlthausen RS, Willrich RM, Kaercher A et al. Fatores associados à não realização de exame citopatológico de colo uterino no extremo Sul do Brasil. Cad Saúde Pública 2003; 19:1365-72.

17. Dias-da-Costa JS, Olinto MTA, Gigante DP, Menezes AMB, Macedo S, Borba AT, et al. Cobertura do exame citológico na Cidade de Pelotas, Rio Grande do Sul, Brasil. Cad Saúde Pública 2003; 19:191-7.

18. Hewitt M, Devesa SS, Breen N. Cervical cancer screening among U.S. women: analyses of the 2000 National Health Interview Survey. Prev Med 2004; 39:270-8.

19. Coughlin SS, Uhler RJ. Breast and cervical screening practices among hispanic women in the United States and Puerto Rico, 1998-1999. Prev Med 2002; 34:242-51.

20. Montano DE, Phillips WR. Cancer screening by primary care physicians: a comparison of rates obtained from physician self-report, patient survey, and chart audit. Am J Public Health 1995 85:795-800.
21. Brenna SMF, Hardy E, Zeferino LC, Namura I. Conhecimento, atitude e prática do exame de $\mathrm{Pa}$ panicolaou em mulheres com câncer de colo uterino. Cad Saúde Pública 2001; 17:909-14.

22. Brasil. Decreto no 94.406/87. Regulamenta a Lei no 7.498 , de 25 de junho de 1986, que dispõe sobre o exercício da Enfermagem, e dá outras providências. Diário Oficial da União 1987; 9 ago.

23. Quadros CAT, Victoria CG, Costa JSD. Coverage and focus of a cervical cancer prevention program in southern Brazil. Rev Panam Salud Publica $2004 ; 16: 223-32$.

24. Leal MC, Gama SGN, Cunha CB. Desigualdades raciais, sociodemográficas e na assistência ao prénatal e ao parto, 1999-2001. Rev Saúde Pública 2005; 39:100-7.

25. Instituto Nacional de Câncer. Controle do câncer de mama. Documento de consenso. Rio de Janeiro: Instituto Nacional de Câncer; 2004.

26. Almeida SDM, Barros MBA. Eqüidade e atenção à saúde da gestante em Campinas (SP), Brasil. Rev Panam Salud Publica 2005; 17:15-25.

Recebido em 04/Mai/2005

Versão final reapresentada em 16/Mar/2006 Aprovado em 17/Mar/2006 